right lateral wall was achieved in all patients. All tachycardias, including scar-related AT and typical AFL were ablated successfully. There was no complication during procedure. No recurrences of AT was observed during follow-up.

Conclusion Under conventional electrophysiological mapping, linear ablations from scar area to anatomic barrier and/or isolation of scar area from right lateral wall, could be successfully abolish IARTs and all potential circuits with reentry around or related to the scar.

\section{e0714 AUTONOMIC NERVE REMODELLING OF PROLONGED RAPID ATRIAL PACING-INDUCED ATRIAL FIBRILLATION AND REVERSAL EFFECT OF PROPRANOLOL}

doi:10.1136/hrt.2010.208967.714

Yang Yu, Li Liu, Jiu Yang Jiang. The First Hospital of Harbin Medical University

Background Limited study disclosed atrial heterogeneous sympathetic innervation in atrial fibrillation recently. But the changes of vagal nerve and the effect of medicine on neural remodelling are not clear.

Methods and Results Twelve dogs underwent atrial pacing at $600 \mathrm{bpm}$. Six of them were given propranolol (1 mg/ $\mathrm{kg}$ tid po, propranolol group), while the other six took placebo only. (AF group) All paced dogs developed sustained AF by 5 weeks of pacing. Tissues from six healthy dogs were used as controls. Immunohistochemistry staining of cardiac nerves was performed using anti-tyrosine hydroxylase $(\mathrm{TH})$ and anti-acetylcholine (Ach) antibodies. In AF group, the density of TH-positive in the right atrium (RA), atrial septum (AS) and left atrium (LA) was $2156.66 \pm 677.57 \mu \mathrm{m}^{2}$ per $\mathrm{mm}^{2}$, $3679.92 \pm 1951.41$ and $18961.8 \pm 10274.15 \mu \mathrm{m}^{2}$ per $\mathrm{mm}^{2}$ respectively, which was significantly $(p<0.01)$ higher than the nerve density in control tissues $(608.8 \pm 414.60,660.62 \pm 273.29$ and $792.46 \pm 326.33 \mu \mathrm{m}^{2}$ per $\mathrm{mm}^{2}$, respectively). In propranolol group, the density of TH-positive nerve in RA, AS, LA was $879.96 \pm 755.13 \mu \mathrm{m}^{2}$ per $\mathrm{mm}^{2}, 960.48 \pm 919.92$ and $1151.98 \pm 678.43 \mu \mathrm{m}^{2}$ per $\mathrm{mm}^{2}$ respectively, which was lower than the AF group. $(p<0.01)$ In AF group, the density of ACh-positive nerve in the RA, AS and LA was $721.94 \pm 341.07 \mu \mathrm{m}^{2}$ per $\mathrm{mm}^{2}, 279.86 \pm 110.25$ and $109.22 \pm 29.86 \mu \mathrm{m}^{2}$ per $\mathrm{mm}^{2}$ respectively, which was significantly $(\mathrm{p}<0.001)$ lower than the nerve density in control tissues (7102.32 \pm 2058.67 , $6093.20 \pm 2512.27$, and $5285.54 \pm 1982.96 \mu \mathrm{m}^{2}$ per $\mathrm{mm}^{2}$, respectively). In propranolol group, the density of ACh-positive nerve in RA, AS, LA was $5356.86 \pm 1733.25 \mu^{2}$ per $\mathrm{mm}^{2}, \quad 4774.86 \pm 1069.30$ and $3550.76 \pm 1399.55 \mu \mathrm{m}^{2}$ per $\mathrm{mm}^{2}$ respectively, which was higher than the AF group. $(p<0.01)$ It proved the truth that higher density of $\mathrm{TH}$ positive nerve $\left(10899.84 \pm 6365.15 \mu^{2}\right.$ per $\left.\mathrm{mm}^{2}, \mathrm{p}<0.001\right)$ and lower density of Ach-positive nerve $\left(127.94 \pm 88.98 \mu \mathrm{m}^{2}\right.$ per $\mathrm{mm}^{2}, \mathrm{P} 2 / \mathrm{mm}^{2}$ $(p<0.01)$ and $4223.22 \pm 1188.23 \mu^{2} / \mathrm{mm}^{2}$ respectively $(p<0.01)$.

Conclusion Sympathetic hyperinnervation and vagal denervation are associated with canine model of sustained AF produced by prolonged left atrial pacing. Propranolol can prevent autonomic remodel to some extent.

\section{e0715 LEFT ANTERIOR DESCENDING ARTERY DISSECTION AND ACUTE MYOCARDIAL INFARCTION AFTER BLUNT CHEST TRAUMA: TWO CASE REPORT}

doi:10.1136/hrt.2010.208967.715

${ }^{1}$ Longbin Liu, ${ }^{1}$ Hangyuan Guo, ${ }^{2}$ Haitao Lv, ${ }^{1}$ Yangbo Xin, ${ }^{1}$ Yufang Qiu, ${ }^{1}$ Fang Pen, ${ }^{1}$ Bio Yang. ${ }^{1}$ Department of Cardiology, Shaoxing People's Hospital, The First Affiliated Hospital of Shaoxing University, Shaoxing City, Zhejiang, China; ${ }^{2}$ Wenzhou Medical College, Wenzhou City, Zhejiang, China

Blunt chest trauma can cause several forms of cardiac injury. Of these, coronary artery dissection and acute myocardial infarction is a rare complication. We report two cases of the left anterior descending coronary artery dissection and acute myocardial infarction after blunt chest trauma.

\section{e0716 AN 81-YEAR-OLD MAN WITH DIZZINESS, FATIGUE AND SHORTNESS OF BREATH}

doi:10.1136/hrt.2010.208967.716

Nan Li. Honorary Clinical Research Associate, Department of Medicine, University of Hong Kong

Case An 81-year-old man presented with dizziness, fatigue and shortness of breath one day after a min-invasive neck spine surgery. The patient said the surgery had been successful, and he was able to get up and walk on the first day, but on the second day he felt dizzy, tired, and dyspnoea. He also found his two hands were swelling. He denied chest pain, unconsciousness, vomiting and other symptoms. The patient was on medication of hypertension and diabetes before the surgery, and both of his wife and son have hypertension. The patient does not smoke nor drink. After surgery, the patient was given antibiotics by venous infusion. On physical examination, the patient looked conscious, well nourished but having a big belly. His temperature was normal. His four extremities were swelling. He had jugular retention. His blood pressure was 164/83 mm Hg, heart rate was $86 \mathrm{bpm}$ and respiratory rate was 24 . Heart auscultation revealed no heart murmur and normal heart rhythm. Pulmonary auscultation found some moist rales at the lower part of two lungs. Chest $\mathrm{x}$-ray showed his heart was boot-shaped, lungs were clear and there was a little pleural effusion in the thorax. Cervical vertebra x-ray showed that the third and fourth spine cords were fixed by steel and there was ossification of nuchal ligament. ECG was almost normal. Echocardiography showed that the internal diameter of left atrium was $50 \mathrm{~mm}$ at diastolic phase; the right atrium was enlarged and the left ventricular posterior wall a bit thicker; the aortic sinus and proximal ascending aorta were widened; the left ventricular ejection fraction was $66 \%$; the diastolic function was slightly reduced. The complete blood count showed: white-cell count $6.11 \times 10^{9} / 1$ neutrophil-cell count $4.22 \times 10^{9} / 1$, percentage of neutrophil-cell $69.1 \%$, red-cell count $4.24 \times 10^{12}$, haemoglobin $125.0 \mathrm{~g} / \mathrm{l}$, haematocrit $37.6 \%$, platelet count $136 \times 10^{9} / 1$. The biochemistry test showed: serum potassium $4.1 \mathrm{mmol} / 1$, serum sodium $142.0 \mathrm{mmol} / \mathrm{l}$, serum chloride $99 \mathrm{mmol} / \mathrm{l}$, carbon dioxide $24.0 \mathrm{mmol} / \mathrm{l}$, serum calcium $2.53 \mathrm{mmol} / \mathrm{l}$, creatinine $124.0 \mathrm{ummol} / \mathrm{l}$, blood urea nitrogen $8.2 \mathrm{mmol} / \mathrm{l}$, uric acid $527.0 \mathrm{umol} / 1$. Serum brain natriuretic peptide level: $348 \mathrm{pg} / \mathrm{ml}$. Blood glucose level: $6.88 \mathrm{mmol} / \mathrm{l}$. The diagnosis of this patient was based on his symptoms of fatigue, and shortness of breath at rest; physical examination of jugular retention, lung rales at lower part and ankle oedema; chest x-ray of pleural effusion. These symptoms and findings indicated the patient had pulmonary congestion and systemic congestion. Though ECG was normal, echocardiography showed diastolic dysfunction, and the serum BNP level was significantly raised. Thus the patient was diagnosed as: acute heart failure, diastolic heart failure, NYHA IV, Stage C; hypertension, grade 3; type 2 diabetes mellitus; cervical spondylopathy, post mini-invasive spine surgery. The treatment included blood pressure monitoring, urinary volume monitoring and electrocardiographic monitoring. Medications included vasodilator nitrate to relief cardiac workload; loop diuretic furosemide to induce diuresis; ACE inhibitor fosinopril to control hypertension; statin atorvastatin to lower lipid level; Insulin to control glucose level. The patient stayed in hospital for 10 days and discharged with no dyspnoea and low extremity oedema, no pulmonary rales, blood pressure $139 / 69 \mathrm{~mm} \mathrm{Hg}$ and heart rate $57 \mathrm{bpm}$. During the 3 months follow-up after discharge, the patient strictly followed doctor's written instructions on diet restriction and exercise. $\mathrm{He}$ measured blood pressure, heart rate and weight and then recorded 
the results every day. His weight maintained 81 to $82 \mathrm{~kg}$. The patient took a walk four times a day and increased from 5 min each time to 15 min each time per day. His medication included diuretic, ACEI, beta-blocker, aspirin, and metformin. Furosemide has been changed to hydrochlorothiazide and maintains at $12.5 \mathrm{mg}$ per day. Aspirin was $100 \mathrm{mg}$ per day. Fosinopril was replaced by valsartan due to dry cough. Valsartan has been gradually up-titrated. When the patient was taking valsartan $80 \mathrm{mg}$ b.i.d., his mean blood pressure was $127 / 67 \mathrm{~mm} \mathrm{Hg}$, but his $24-\mathrm{h}$ ambulatory blood pressure monitoring showed that his night-time blood pressure was nondipping and about $80 \%$ was over $120 / 80 \mathrm{~mm} \mathrm{Hg}$ despite of the well controlled day-time blood pressure. Then valsartan was uptitrated to $80 \mathrm{mg}$, t.i.d., and his morning supine blood pressure lowered from 155/83 $\mathrm{mm} \mathrm{Hg}$ to $132 / 74 \mathrm{~mm} \mathrm{Hg}$ and maintained at about $116 / 60 \mathrm{~mm} \mathrm{Hg}$ during the day. Bisotolol was not up-titrated due to his relative low heart rate and diabetes. Discussion Heart failure is the leading cause of hospitalisation and mortality in developed and developing countries despite of the improvement of heart disease and longevity of population. ${ }^{1}$ It is primarily developed in the elderly population and cause big economic burden to the society. In the United States, the annual incidence of heart failure hospitalisation in patients older than 65 years is 10 per 1000 . The average duration of hospitalisation is about 6 days. About $2 \%$ of hospital admission is the decompensated heart failure and rehospitalisation rate reaches $50 \%$ in 6 months after discharge. Approximately two thirds of cost attributes to the management of episodes of acute decompensated heart failure. The underlined causes of heart failure are coronary artery disease, hypertension, diabetes, metabolic syndrome and others. Though myocardial infarction accounts for $60 \%$ of heart failure, $70 \%$ of heart failure has a medical history of hypertension. Up till now, there are $40 \%$ to $50 \%$ acute decompensated heart failure has unknown causes. The poor compliance with medication or diet restriction, under controlled hypertension or diabetes, ischaemia, anaemia, diabetes, renal dysfunction, drugs such as calcium-channel blockers and nonsteroidal anti-inflammatory drugs may precipitate decompensated heart failure. In patients with hypertension, blood pressure is normally monitored at doctor's office and at patient home. The information of blood pressure may be not complete by day-time office monitoring if the patient has nondipping hypertension or nocturnal hypertension. Studies show that hypertension with nondipping pattern especially those with nondipping diastolic blood pressure contributes to congestive heart failure. The underlined mechanism could be the increased left ventricular filling pressure. ${ }^{2}$ The diagnosis of congestive heart failure is based on suspected symptoms such as shortness of breath at rest or exertion, fatigue or tiredness and oedema at ankle; typical signs on physical examination such as tachycardia, pulmonary rales, raised jugular venous pressure, peripheral oedema, cardiomegaly, abnormal murmur and hepatomegaly; specific test results such as normal or abnormal ECG, echocardiogram with left ventricular enlargement and low left ventricular ejection fraction, and elevated serum brain natriuretic peptide level. The symptoms of heart failure indicate the deficiency of blood supply of tissues and organs due to the pump failure. ECG may be normal or abnormal due to ischemia. If echocardiogram shows the left ventricular enlargement and reduced left ventricular ejection fraction, it indicates the patient has systolic heart failure; if echocardiogram shows preserved left ejection fraction but left atrium enlargement and reduced diastolic function, it indicates the patient has diastolic heart failure. Serum brain natriurectic peptide level may increase due to increased left ventricular filling pressure, when its level surpasses $100 \mathrm{pg} / \mathrm{ml}$ heart failure is considered. Elevated blood natriuretic peptide levels (BNP or NT-proBNP) help to differentiate other diseases such as COPD, pulmonary embolism and pneumonia. The exercise capacity may be evaluated by the $6 \mathrm{~min}$ walk test. Heart failure is currently classified into four stages: Stage A-high risks of heart failure; Stage B-abnormal cardiac structure without symptoms; Stage C-abnormal cardiac structure with symptoms; Stage D-refractory symptoms requiring specialised, advanced treatment strategies. For a patient diagnosed with heart failure, prognostic stratification of heart failure should be evaluated, as the mortality of heart failure is high, especially in elderly patients. NYHA functional classification used to be the most common standard for the severity of heart failure, however it is subjective under doctor's judgement. Symptoms, though relate to the severity of heart failure, should not be used to guide drug therapies, especially titration of ACE-inhibitors or beta-blockers. Staging classification provides objective scales to heart failure and clinical trial showed heart failure staging were associated with progressively increasing plasma BNP concentration and progressively higher 5 -year mortality rates. The plasma level of natriuretic peptides is significantly related to the severity of heart failure. It is used as the surrogate marker of heart failure. ${ }^{3}$ High plasma BNP level is associated with high risk of rehospitilization and death. A plasma level of $\mathrm{BNP}>1000 \mathrm{pg} / \mathrm{ml}$ despite of optimal treatments usually indicates a poor prognosis. The treatment of chronic heart failure at stage $C$ aims to prevent progression and cardiac events. Organising a heart failure team is effective to manage chronic heart failure patients. Education and instruction on diet, exercise, weight control and early sign of decompensation to patients should be done before dischargement or right after diagnosis of heart failure during out-patient clinic. Follow up of patients should be conducted by any possible method such as telephone and appointment. Each patient is encouraged to self care of the disease at home. Patients shall be educated at discharge that some factors may precipitate heart failure decompensation such as poor compliance with medication or dietary restriction, ischemia, hypertension, and arrhythmias. The other factors are anaemia, diabetes, renal dysfunction and medications such as calcium-channel blockers and nonsteroidal anti-inflammatory drugs. Besides education, written instruction with core content of follow up shall be provided to patients, including recommended exercise frequency, weight variation, medication and up-titration. During the follow-up, a well designed checklist including blood pressure, blood glucose, heart rate, weight, exercise frequency and time, change of medications or therapy, and careplayer telephone number is recommended to be provided to heart failure patients for his/her selfcare management. Detailed and insistent records help doctor to understand the patient process thoroughly. Early detection of congestion is critical for treatment adjustment so as to prevent symptoms and rehospitalization. Beside daily weight monitoring by patients themselves, an intrathoracic impedance device is under investigated and promising results show that the device will be superior to daily weight monitoring to alarm the haemodynamic congestion of heart failure. ${ }^{4}$ Similarly, monitoring of serum BNP level is also an effective approach in clinic to predict the episodes of worsening of heart failure. 